\title{
ORIGINAL
}

\section{FACTORES ASOCIADOS CON LA COBERTURA VACUNAL DECLARADA EN LA PRIMERA INFANCIA: RESULTADOS DE UNA ENCUESTA TELEFÓNICA $\left({ }^{*}\right)$}

\author{
Manel Nebot (1), Enrique Muñoz (1), Marta Figueres (2) Gloria Rovira (1), Montserrat Robert (1) \\ y Dolors Minguell (1). \\ (1) Institut Municipal de Salut Pública, Barcelona. \\ (2) Institut de Salut Pública de Catalunya. \\ (*) Estudio parcialmente financiado con una ayuda del Fondo de Investigaciones Sanitarias (97/0660)
}

\section{RESUMEN}

Fundamentos: El Plan de Vacunación Continuada de Barcelona permite conocer la cobertura vacunal poblacional a través de un sistema de declaración postal voluntaria de las familias. Estudios anteriores han demostrado que existe una proporción importante de familias que no notifican la vacunación, lo que puede conducir a una infraestimación de la cobertura real. Los objetivos de este estudio son estimar la cobertura vacunal poblacional en la primera infancia y conocer los factores asociados con la no declaración de la vacunación.

Métodos: Se realizó un cuestionario telefónico a una muestra de 500 niños de los que no constaba la declaración de las tres primeras dosis de vacunación en la infancia (difteria, tétanos, tos ferina y polio oral), así como a una muestra de 500 niños que constaban como vacunados. Para estimar la cobertura vacunal real se consideró correctamente vacunados a todos los niños cuando así lo declaraban sus familiares. Con respecto a las no respuestas, se consideró en el caso más desfavorable que correspondían a niños no vacunados. En la situación más favorable, se atribuyó una cobertura similar a la de las respuestas.

Resultados: La respuesta al cuestionario fue superior entre los que habían declarado previamente la vacunación a través del sistema de notificación postal $(79,1 \%)$ que entre los que no la habían notificado $(67 \%)$ ). Entre los factores que se asociaron a la no declaración del estado vacunal destaca el tamaño de las familias, la utilización de servicios sanitarios privados, y el lugar de nacimiento de los padres. Unicamente 6 casos entre los que no habían notificado la vacunación admitieron no haber vacunado a sus hijos, lo que representa un $1,9 \%$ de las respuestas. La cobertura vacunal poblacional sería del $99,7 \%$ en el caso más favorable y del $93,7 \%$ en la situación menos favorable.

Conclusiones: La cobertura vacunal poblacional es muy elevada. Los resultados subrayan la importancia de promover la notificación de la vacunación entre los profesionales sanitarios, especialmente en el sector privado.

Palabras clave: Vacunas. Infancia. Cataluña. Monitorización. Encuesta telefónica. Cobertura vacunal.

Correspondencia:

Manel Nebot

Servicio de Promoción de la Salud.

Institut Municipal de Salut Pública.

Pl. Lesseps 1

08023 Barcelona

Correo electrónico: mnebot@imsb.bcn.es

\section{ABSTRACT}

\section{Factors Associated with Reported Childhood Immunization Coverage. Results of a Telephone Survey}

Background: Barcelona's Continuing Immunization Plan affords the possibility Of monitoring the immunization coverage of the population by means of the voluntary family postal notification system. Prior studies have revealed that some families fail to provide notification while being correctly vaccinated, which can lead to actual coverage being underestimated. The objectives of this study are to estimate the early childhood immunization coverage of the population and to ascertain the factors associated with failure to provide notification of immunization.

Methods: A phone survey was conducted on a sample of 500 children regarding whom there was no record of any notification of the first three childhood vaccine doses (diphtheria, tetanus, whooping cough and oral polio), in addition to a sample of 500 children who were on record as having been immunized. To estimate the actual immunization coverage, all children were considered to have been properly immunized when their family members did provide notification. As regards those who failed to reply, it was considered in the worst of cases that these were cases of children who had not be immunized. In the best of cases scenario, a coverage similar to those of the responses was assumed.

Results: The response to the questionnaire was higher among those who had previously provided notification of immunization by way of the postal notification system $(79,1 \%)$ than among those who had failed to provide notification of immunization $(67 \%)$. The leading factors associated with failure to report immunization status were the size of the families, the use of private health care services and the place of birth of the parents. Solely six (6) cases of those who had failed to report immunization admitted to not having immunized their children, totaling $1,9 \%$ of the responses. The immunization coverage of the population in question would total $99,7 \%$ in the best of cases and $93,7 \%$ in the worst of cases scenario.

Conclusions: Immunization coverage of the population in question is quite high. The results underline the importance of promoting immunization notification among health care professionals, especially in the private sector.

Key words: Immunization; coverage. Monitoring. Telephone survey. 


\section{INTRODUCCIÓN}

Las vacunas o inmunizaciones activas ocupan un lugar muy destacado en la prevención de las enfermedades transmisibles, especialmente en aquéllas en las que el reservorio es humano y cuando la transmisión es interhumana ${ }^{1}$. En estas enfermedades las vacunaciones no sólo proporcionan protección individual sino también una protección colectiva o comunitaria que contribuye a romper la cadena epidemiológica de contagio y permite obtener una protección superior a la suma de las inmunidades individuales ${ }^{2}$. En la actualidad, la cobertura vacunal en la infancia es muy elevada en la mayoría de los países desarrollados; así, en la región europea la cobertura de la inmunización primaria con tres dosis de vacuna contra la difteria, tétanos y tos ferina (DTP) y polio oral es superior al 90\%, con independencia del sistema sanitario y de la estrategia vacunal ${ }^{3}$, mientras que en los Estados Unidos la cobertura en 1999 era del 89,6\% ${ }^{4}$.

En nuestro país, las vacunaciones han sido impulsadas históricamente desde los departamentos locales de salud pública $\mathrm{y}$ más recientemente por las comunidades autónomas. En Barcelona existe desde principios de siglo un sistema de registro de las vacunaciones basado en la notificación por las familias. En 1973 este registro fue informatizado, al tiempo que se estableció un sistema de tarjetas pre-franqueadas que se enviaban al domicilio de todos los recién nacidos junto con el carnet vacunal. Estas tarjetas permitían la notificación postal progresiva del cumplimiento de las distintas etapas del calendario vacunal ${ }^{5,6}$. Este sistema de registro permite monitorizar la cobertura vacunal así como certificar en cualquier momento el estado vacunal de un ciudadano que lo precise. Sin embargo, infraestima la cobertura real al registrar únicamente vacunación notificada. Así, mientras la cobertura vacunal declarada para la primovacunación con tres dosis de vacuna DTP (difteria, tétanos y pertussis) oscila alrededor del $80 \%$ en los últimos años, diversos estudios en determinadas zonas de la ciudad y en algunos co- lectivos específicos han puesto de manifiesto que la mayoría de casos en que la tarjeta de notificación no es enviada para su registro corresponden a olvido o extravío de las mismas $^{7,8}$. Para estimar la magnitud y las causas de la no notificación en una muestra representativa de la población se diseñó el presente estudio, cuyos objetivos son estimar la cobertura vacunal en los niños cuyas familias no han devuelto la tarjeta de notificación, y explorar los factores asociados a la falta de notificación.

\section{MATERIAL Y MÉTODOS}

En mayo de 1997 se seleccionó del registro de vacunaciones del Instituto Municipal de Informática una muestra de 500 niños entre 2 y 3 años (nacidos entre el 20.05.94 y el 19.05.95) que constaban como no vacunados en el registro de vacunaciones, al no haberse recibido la tarjeta de notificación de la primovacunación (T-1). Como grupo de control se eligió una muestra formada por 500 niños que constaban como vacunados según el mismo registro. Por errores en los datos o por cambio de domicilio fueron eliminados 33 casos, resultando finalmente en una muestra de 967 casos, 488 que habían enviado la T-1 y 479 que no la habían enviado.

Para el seguimiento telefónico se diseñó un cuestionario, basado en parte en cuestionarios previos ${ }^{9}$, en el que se determinaban el recuerdo del profesional o centro donde se había realizado la vacunación, el tipo de asistencia sanitaria habitual, el tamaño y composición de la familia, y determinadas creencias relacionadas con las vacunaciones y sus contraindicaciones. A efectos de estimar la cobertura vacunal poblacional se ha considerado como correctamente vacunados a todos los niños de los que se había recibido notificación postal de la primovacunación (T-1). Con respecto a las familias que no habían enviado la tarjeta, se consideraron como bien vacunados todos los que lo declaraban; finalmente, en relación con las no respuestas, se consideraron dos escenarios posibles, que re- 
presentan los límites superior e inferior de la cobertura estimada: en el escenario más favorable, se consideró que las no respuestas se comportarían como las respuestas, y por tanto se les atribuyó la misma proporción de vacunados; por el contrario, en el escenario más desfavorable se consideró que todas las no respuestas corresponderían a niños no vacunados.

El nivel socioeconómico fue asignado a partir del Indice de Capacidad Económica Familiar, índice sintético de diversos indicadores económicos ampliamente utilizado en estudios previos en Barcelona ${ }^{10}$.

La entrevista fue realizada personalmente por dos de las autoras del estudio (MF y GR), siguiendo un protocolo de llamadas a horas diversas (un mínimo de 5 llamadas cubriendo las franjas horarias de mañana, mediodía, tarde y noche), y después de una prueba piloto en una submuestra de domicilios. En todos los casos se solicitaba la respuesta de la madre, el padre o algún otro familiar directo, por este orden.

\section{RESULTADOS}

Tras la eliminación de datos de identificación incompletos o erróneos se obtuvo finalmente una muestra de 967 casos, 488 que ha- bían enviado la T-1 y 479 que no la habían enviado. Se obtuvo respuesta al cuestionario en 707 (73,1\% del total), principalmente de la madre $(82,7 \%)$, seguida de otros familiares, fundamentalmente el padre $(16,0 \%)$. En la tabla 1 se presentan las características socioeconómicas de la muestra según la notificación previa del estado vacunal (devolución de la tarjeta T-1) y la respuesta al cuestionario. La proporción de respuestas fue superior entre las familias que habían notificado la primovacunación, siendo la diferencia estadísticamente significativa. El resto de variables demográficas (edad media, sexo y nivel socioeconómico del barrio de residencia) no mostraron diferencias significativas.

En la tabla 2 se presenta la distribución de diversas variables en relación con la notificación de la primovacunación. El número de niños y el número total de personas conviviendo en el domicilio, así como el lugar de nacimiento de al menos uno de los dos progenitores fuera de Barcelona, se asociaron negativamente a la notificación postal de la inmunización, mientras que la utilización habitual de servicios sanitarios públicos se asoció con una mayor probabilidad de la notificación. De las dos variables de tamaño familiar, en el análisis multivariado únicamente se mantuvo la asociación de la no declaración con el número total de perso-

Tabla 1

Características socioeconómicas de la muestra según notificación previa del estado vacunal y la respuesta al cuestionario

\begin{tabular}{|c|c|c|c|}
\hline & \multicolumn{3}{|c|}{ Primovacunación notificada } \\
\hline & Si $(N=488)$ & No $(N=479)$ & Total $(N=967)$ \\
\hline Respuesta al cuestionario (n; \%) & $386(79.1 \%)$ & $321(67.0 \%)$ & $707(73.1 \%)^{*}$ \\
\hline Edad (media; IC) & $2.54(2.52-2.56)$ & $2.52(2.49-2.54)$ & $2.53(2.51-2.55)$ \\
\hline N.o llamadas (media; IC) & $3.61(3.43-3.79)$ & $3.70(3.50-3.89)$ & $3.65(3.52-3.78)$ \\
\hline Sexo (n; \% de niñas) & $215(44.1 \%)$ & $226(47,2 \%)$ & $441(45.6 \%)$ \\
\hline \multicolumn{4}{|l|}{ Nivel socioeconómico: } \\
\hline Bajo & $129(26.4 \%)$ & $124(25.9 \%)$ & $253(26.2 \%)$ \\
\hline Medio & $207(42.4 \%)$ & $210(43.8 \%)$ & $417(43.1 \%)$ \\
\hline Alto & $152(31.2 \%)$ & $145(30.3 \%)$ & $297(30.7 \%)$ \\
\hline
\end{tabular}

(*) $\mathrm{p}<0.0001$.

(a): basado en índice de capacidad económica familiar (ICEF). 
Tabla 2

Variables asociadas con la no declaración postal de la primovacunación

\begin{tabular}{|c|c|c|c|c|}
\hline & \multicolumn{2}{|c|}{ Notificación postal } & \multirow{2}{*}{$p$} & \multirow{2}{*}{ ORa (IC 95\%) } \\
\hline & $\operatorname{Si}(N=386)$ & No $(N=321)$ & & \\
\hline Padre o /y madre nacidos fuera de Barcelona $(\% ; n)$ & $42,0 \%(162)$ & $49,5 \%(159)$ & 0,05 & $1,34(0,99-1,80)$ \\
\hline Número de niños conviviendo en el domicilio (media; IC)\$ & $2,75(2,68-2,82)$ & $2,95(2,84-3,06)$ & 0,007 & $1,74(1,16-2,63)$ \\
\hline $\begin{array}{l}\text { Número total de personas conviviendo en el domicilio (me- } \\
\text { dia; IC)\& }\end{array}$ & $3,79(3,71-3,87)$ & $4,95(3,93-4,05)$ & 0,0007 & $1,94(1,32-2,85)$ \\
\hline $\begin{array}{l}\text { Número total de personas conviviendo en el domicilio en } \\
\text { situación de desempleo (media; IC)\# }\end{array}$ & $0,19(0,17-0,21)$ & $0,18(0,16-0,20)$ & NS & $0,96(0,65-1,42)$ \\
\hline Utilización habitual de servicios sanitarios públicos (\%; n) & $43,1 \%(166)$ & $32,7 \%(104)$ & 0,005 & $1,55(1,14-2,11)$ \\
\hline Continuidad de la asistencia con el mismo pediatra $(\% ; n)$ & $73,1 \%(282)$ & $73,2 \%(235)$ & NS & $1,00(0,72-1,40)$ \\
\hline
\end{tabular}

ORa: odds ratio ajustado.

\$: categoría de referencia en el análisis de regresión logística: tres o menos (vs 4 o más)

\& categoría de referencia en el análisis de regresión logística: hasta cuatro personas (vs 5 o más)

\# categoría de referencia en el análisis de regresión logística: ninguno (vs uno o más)

nas, con un odds ratio (OR) de 2,59, intervalo de confianza del 95\% (IC) de 1,69-3,97, para las familias con 5 o mas personas. Así mismo, en el análisis multivariado se asociaron significativamente con la notificación la utilización regular de los servicios sanitarios públicos $\left(\mathrm{OR}=1,71 ; \quad \mathrm{IC}_{95 \%}\right.$ $1,44-2,02)$ y la procedencia de uno de los padres de fuera de Barcelona (OR 1,37; $\mathrm{IC}_{95 \%}$ 1,17-1,60).

En la tabla 3 se presenta el estado vacunal declarado en los dos grupos estudiados. Entre los que notificaron por correo la primovacunación el 91,5\% recordaba el centro o profesional responsable de la vacunación. Entre los que no habían enviado la tarjeta de notificación la proporción que recordaba con precisión la administración de las vacunas o tenía algún registro escrito era similar $(89,7 \%)$, pero había 6 casos $(1,9 \%)$ que declaraban no haber administrado las vacunas por motivos diversos, en su mayoría relacionados con las creencias de los padres contrarias a las vacunas.

En la figura 1 se presenta la estimación de la cobertura vacunal poblacional en los dos escenarios de atribución de proporción de niños correctamente vacunados en las no respuestas. En el escenario más favorable, considerando que las no respuestas se comportarían igual que los que responden, la proporción de vacunados en el grupo que no ha notificado la vacunación sería la observada entre los que responden a la entrevista $(98,1 \%)$, lo cual supone una cobertura global del 99,7\%. En cambio, en el escenario

Tabla 3

Estado vacunal declarado según la notificación postal de la primovacunación

\begin{tabular}{|lccc|}
\hline \multirow{2}{*}{$\begin{array}{c}\text { Notificación postal previa } \\
\text { de la primovacunación }\end{array}$} & \multicolumn{3}{c|}{ Estado vacunal declarado } \\
\cline { 2 - 4 } & $\%(n)$ & No recuerdan profesional o centro & Recuerdan profesional o centro \\
\hline & $0,0(0)$ & $\%(n)$ & $\%(n)$ \\
\hline Sí $(\mathrm{N}=386)$ & $1,9 \%(6)$ & $8,5 \%(33)$ & $91,5 \%(353)$ \\
No $(\mathrm{N}=321)$ & & $8,4 \%(27)$ & $89,7 \%(288)$ \\
\hline
\end{tabular}


Figura 1

Cobertura vacunal estimada en los dos escenarios de atribución del estado vacunal de las no respuestas. Barcelona, 1997-1998

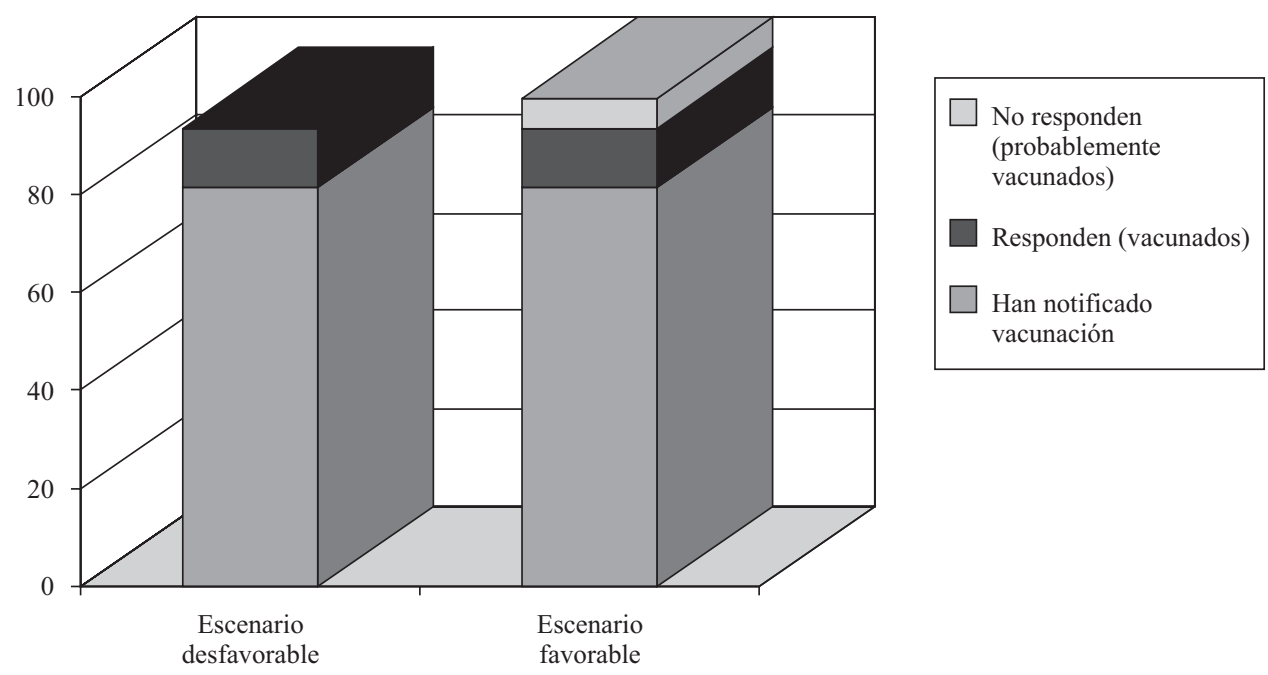

más desfavorable, la proporción de niños correctamente vacunados entre los que no han notificado la primovacunación es del $65,7 \%$, y la proporción poblacional estimada es del 93,6\%.

\section{DISCUSIÓN}

Los resultados del estudio muestran una respuesta al cuestionario telefónico aceptable $(73 \%)$, aunque cabe señalar un claro sesgo de respuesta, ya que ésta fue más frecuente entre los que habían retornado la tarjeta de notificación de las vacunas. Entre las variables asociadas con la notificación, el mayor tamaño de las familias y el lugar de nacimiento fuera de la ciudad se asocian con una menor probabilidad de declarar el estado vacunal, mientras que el uso de servicios sanitarios públicos se relaciona con una mayor notificación. Aunque el desconocimiento de la situación vacunal de las no respuestas impide conocer con exactitud la cobertura real poblacional, los resultados apuntan un límite inferior $(93,7 \%)$ ciertamente satis- factorio, que alcanzaría para estas enfermedades vacunables los niveles deseables de inmunidad de grupo, en consonancia con los objetivos del Plan de Vacunación Continuada de la ciudad ${ }^{11}$.

Entre las limitaciones del estudio cabe señalar en primer lugar el potencial sesgo de respuesta. Si globalmente la tasa de respuesta $(73 \%)$ puede considerarse satisfactoria en un cuestionario telefónico, y es superior a la que se obtiene habitualmente por otros métodos como las encuestas por correo ${ }^{11}$, la mayor proporción de no respuestas entre las familias que no habían notificado la vacunación podría reflejar diferencias más globales, que podrían traducirse en un comportamiento distinto en relación a las inmunizaciones. Para minimizar los efectos de este sesgo se han considerado dos escenarios de atribución del comportamiento de las no respuestas, tomando como hipótesis que las familias que no responden tienen una menor cobertura vacunal que las que si lo hacen. En este sentido, existen suficientes evidencias de que las no respuestas tienen en gene- 
ral comportamientos mas desfavorables ${ }^{12}, \mathrm{y}$ también de que la cobertura vacunal es menos elevada entre los grupos socioeconómicos menos favorecidos ${ }^{13-15}$. Sin embargo, es improbable que todas las no respuestas correspondan a niños no vacunados. De hecho, en el estudio de Vigilancia de la Inmunización de Estado Unidos, en el que se realiza una encuesta telefónica a una muestra de 33.000 familias, el sesgo a la baja debido a la no respuesta telefónica se estima en tan solo el $1,3 \%{ }^{16}$. Por otro lado, los estudios seroepidemiológicos realizados en nuestro medio apuntan en general a coberturas vacunales muy elevadas ${ }^{17,18}$, al igual que las encuestas sistemáticas a las familias al inicio de la escolarización ${ }^{7}$.

Con respecto a la validez del método para atribuir el estado vacunal a través de la declaración telefónica, y ante la imposibilidad de disponer de un estándar inequívoco de comparación, como sería la observación directa del carnet vacunal o de algún registro escrito, se ha asumido como válida la declaración de los padres, aún en el caso de que no recordaran el centro o profesional, dado que la proporción que no podía recordar este dato era la misma en el grupo que había notificado la vacunación -certificada por un profesional sanitario- $-\mathrm{y}$ en el que no lo había hecho, lo que puede ser debido al tiempo transcurrido entre la primovacunación y la encuesta, en algunos casos superior a dos años. De hecho, la encuesta telefónica es una entre las opciones posibles para monitorizar la cobertura vacunal ${ }^{6}$, si bien su fiabilidad y bajo coste la convierten en uno de los métodos más fiables, siendo utilizado actualmente para monitorizar la cobertura vacunal en Estados Unidos ${ }^{16}$.

Entre los factores asociados a la no declaración de la vacunación, la menor probabilidad de recibir determinados cuidados, entre los que se incluyen las vacunas, a medida que aumenta el número de hijos ha sido descrito en anteriores estudios ${ }^{19,20}$. La mayor notificación entre las familias que utilizan los servicios sanitarios públicos puede indicar un mejor conocimiento del sistema de información del carnet de vacunas entre estos profesionales, y debería ser objeto de un análisis más profundo que permitiera establecer mecanismos de corrección. El lugar de nacimiento de los padres como factor de riesgo para la no declaración podría deberse a su correlación con el nivel socioeconómico tanto como al desconocimiento de los procesos administrativos de la ciudad, aunque los resultados no permiten decantarse hacia ninguna de las hipótesis.

El estudio ha permitido obtener una aproximación fiable al comportamiento vacunal de las familias que no notifican la tarjeta vacunal, y sus resultados apuntan hacia la necesidad de sensibilizar a los pediatras, especialmente en el sector privado, de la importancia de la notificación, evitando que sea percibida como un simple trámite administrativo. También sabemos que hay una proporción muy pequeña que manifiestan abiertamente no estar vacunados; probablemente es un indicador ante el que deberemos estar alertas en el futuro. Con todo, permanece la duda acerca del estado vacunal de una parte de la población, que no notifica el estado vacunal y no ha respondido al cuestionario, lo que aconseja combinar todas las fuentes de datos fiables de las que se disponga para obtener una aproximación fiable al grado de cobertura real.

\section{BIBLIOGRAFÍA}

1. Salleras L. Concepto, clasificación y características generales de las vacunas. En: Salleras L Vacunaciones preventivas. Principios y aplicaciones. Barcelona: Masson; 1998, p. 3-14.

2. Vaqué J. Inmunidad colectiva o de grupo. En: Salleras L Vacunaciones preventivas. Principios y aplicaciones. Barcelona: Masson; 1998, p. 3-14.

3. Roure C. Situación de los programas de inmunización en la Región Europea de la OMS. En: Salleras L Vacunaciones preventivas. Principios y aplicaciones. Barcelona: Masson; 1998, p. 3-14.

Rev Esp Salud Pública 2001, Vol. 75, N. ${ }^{\circ} 1$ 
4. Mortality and Morbidity Weekly Report. National, state and urban area vaccination coverage levels among children aged 19-35 months. United States 1999. MMWR 2000; 43: 585-589.

5. Palomar M. El procedimiento administrativo del plan de vacunación continuada. Boletín de la Sociedad Catalana de Pediatría 1974; 35: 116-144.

6. Villalbí JR. La cobertura vacunal en la infancia. Rev Salud Pública 1991; 2: 89-105.

7. Albertos A, Rovira G, Villalbí JR. Cobertura vacunal en escolares. Rev San Hig Púb 1991; 65: 269-75.

8. Villalbí JR, Graugés MD. Cobertura vacunal a los dos años de edad: un estudio transversal en la ciudad de Barcelona. An Esp Pediatr 1990; 32 (4): $321-24$.

9. Colomer C, Tuells J, Nolasco A, Bolumar F, Alvarez-Dardet C. Conocimientos y creencias en relación con las enfermedades vacunables y su prevención. Estudio en la comunidad de Elche. Med Clin (Barc) 1987; 89 (7): 275-277.

10. Ventura A, Cárcel C, García R, Pujol C, Tomás P, Aluja T, Rius R, Vilagut G. Indice de Capacidad Económica Familiar en Barcelona II. Barcelona: Publicaciones del Ayuntamiento de Barcelona; 1999.

11. Institut Municipal d'Higiene, Ajuntament de Barcelona. Pla de Vacunació continuada de la població infantil de Barcelona. Barcelona: Ajuntament de Barcelona, Publicacions de Salut Pública; 1978 .

12. Frey JH. Survey research by telephone. Newbury Park (CA): Sage Publ; 1989.
13. Ganduxer D, Villalbí JR. Asistencia médica y cobertura vacunal de los hijos de mujeres filipinas. An Esp Pediatr 1993; 39 (3): 235-239.

14. García E, Vioque J. Cobertura vacunal en los distritos de la ciudad de Castellon y su relación con factores sociodemográficos: un estudio ecológico. Med Clin 1993; 101: 333-7.

15. Villalbí JR, Díez E, Foz M, Graugés MD, Rovira G, Massip M. Cobertura vacunal a los dos años de edad en una zona socialmente desfavorecida. Aten Prim 1992; 9(3): 154-56.

16. Zell ER, Ezatti-Rice TM, Battaglia MP, Wright RA. National Inmunization Survey: the methodology of a vaccination surveillance system. Pub Health Rep 2000; 115: 65-77.

17. Salleras L, Vidal J, Canela J, Jiménez de Anta MT, Pumarola T, Coll JJ, De la Puente ML, Serra L. Seroepidemiology of measles in Catalonia (Spain) 1985-1986. Eur J Epidemiol 1990; Jun 6 (2): 207-211.

18. Vidal J, Canela J, de la Puente ML, Jiménez de Anta MT, Pumarola T, Coll JJ, Salleras L. Prevalence of antitetanus antibodies in pregnant women from Catalonia Enferm Infecc Microbiol Clin 1989 Apr; 7 (4): 203-205.

19. Barreto TV, Rodríguez LC. Factors influencing childhood immunization in an urban area in Brazil. J Epidemiol Com Health 1992; 46: 357-61.

20. Beltrán JL, Lucas R, Martínez JM, Martínez F. Cobertura vacunal infantil en una comunidad rural: prevalencia y factores asociados. Gac San 1990; 4 (17): 60-6. 